\title{
Impact of the COVID-19 Pandemic \& Telehealth Implementation in a Student Run Free Clinic
}

\author{
Michela M. Fabricius $^{1}$ (1) $\cdot$ Nicole M. Hitchcock ${ }^{1} \cdot$ Zachary C. Reuter $^{1} \cdot$ Madeline E. Simon $^{1} \cdot$ Robert P. Pierce $^{2}$
}

Accepted: 12 September 2021 / Published online: 22 September 2021

(c) The Author(s), under exclusive licence to Springer Science+Business Media, LLC, part of Springer Nature 2021

\begin{abstract}
Student run free clinics (SRFCs) fill a void in healthcare access for many communities and have been subject to unprecedented shifts in care delivery brought about by the coronavirus disease 2019 (COVID-19) pandemic. Our single-center institution serving uninsured patients in central Missouri switched from in-person visits to strictly telehealth visits with the onset of the pandemic. This study investigated the impact of the pandemic and the switch to telehealth on the clinic return rates by ethnicity, race, gender, rurality, and age. The pandemic led to a $47.4 \%$ reduction in the number of monthly patient encounters. Of the established SRFC population $(\mathrm{N}=309)$, only 87 patients $(28.2 \%)$ returned for a telehealth visit during the COVID-19 pandemic. Older patients ( $\geq 45$ years old) were more likely to return (OR 1.71, 95\% CI 1.02-2.85) for care via telehealth after the onset of the pandemic than younger patients ( $<45$ years old). No differences in the likelihood of returning for a telehealth visit were identified by race, ethnicity, gender, or rurality. Telehealth offers an effective solution to the complex problems faced by SRFCs during the COVID-19 pandemic and has not added barriers to care with regards to race, ethnicity, gender, or rurality at our SRFC.
\end{abstract}

Keywords COVID-19 pandemic $\cdot$ Uninsured $\cdot$ Health disparities $\cdot$ Telehealth $\cdot$ Student run free clinic

\section{Introduction}

The coronavirus disease 2019 (COVID-19) pandemic has had far-reaching impacts on public health, economic, and social landscapes globally. The United States alone is steadily nearing 39 million confirmed cases with more than 620,000 COVID-19 attributable deaths as of mid-2021 [1]. Additionally, it is well known that the pandemic has exacerbated health disparities, particularly racial and ethnic ones [2-6]. Black patients are more likely to have health risks associated with severe COVID-19 illness as compared with White patients [2]. Cumulative infection, death, and hospitalization rates are higher among historically underrepresented racial/ethnic groups than among White patients, especially in those 35 years of age and older [5].

Michela M. Fabricius

Mmtcd6@health.missouri.edu

1 University of Missouri School of Medicine, One Hospital Drive, Columbia, MO, USA

2 Department of Family and Community Medicine, University of Missouri, Columbia, MO, USA
In Missouri, Medicaid expansion has not yet been implemented, which renders many individuals vulnerable and without healthcare coverage [7, 8]. MedZou Community Health Clinic is a medical student-run free clinic (SRFC) in Columbia, Missouri that helps cover this gap and provide care to the uninsured. On March 17, 2020, the Association of American Medical Colleges issued a joint statement pausing medical student involvement with patient care due to the COVID-19 pandemic [9]. Prior to this date, all MedZou visits were performed in-person. Following the release of this statement, all in-person visits at our single-center institution were suspended, and MedZou shifted to using telehealth as the sole method for patient visits until April 1, 2021.

This change in care delivery was not unique to our free clinic. Rapid implementation and utilization of telehealth services occurred nationally in response to the growing pandemic. This change evolved as an effort to continue providing care to patients, while simultaneously limiting the risk of disease transmission [10-12]. Successful efforts to create and offer telehealth services to uninsured or underinsured patients receiving care from SRFCs nationwide have been described [13-22]. To date, research has focused primarily on the methods of telehealth implementation in SRFCs. No 
work has described the impact of the pandemic by age, sex, race, or ethnicity on patients seeking care at SRFC.

This study aims to determine if and what demographic differences exist (age, sex, race, ethnicity, and rurality) between those MedZou patients who did and did not return to clinic after telehealth implementation during the COVID19 pandemic.

\section{Methods}

This retrospective analysis was performed using data from a single-center, SRFC in the USA (University of Missouri Health System, MedZou Clinic). Using the MU Health Center electronic health record (EHR), patient demographics were examined for those patients seen between March 17, 2020 and April 1, 2021 (during the COVID-19 pandemic) and compared to a sample between August 23, 2018 and March 16, 2020 (pre-pandemic).

A retrospective analysis was performed on patient data for visits before and after the onset of the pandemic. The primary outcome was the likelihood of established patients returning to MedZou for a telehealth encounter during the COVID-19 pandemic. The independent variables included age ( $<45$ years, $\geq 45$ years); sex (male, female); ethnicity (Hispanic, non-Hispanic, other); race (White, Black or African American, other); and rurality. Due to limited sample size, the "other" category for race includes, but is not limited to, patients identifying as Asian, some other race not specified, or patients without a documented race in the health record. Postal codes from reported addresses were used to assign rurality according to the definitions of the Federal Office of Rural Health Policy. All variables considered in the study were self-reported by patients. Secondary analysis compared pre- and post-pandemic return rates. Bivariate analyses were performed using chi-square tests and multivariate analyses were performed using logistic regression. Statistical analysis was performed via the Stata/ IC v16.0 (College Station, TX) software, with a p-value of 0.05 determined to be statistically significant. This study was reviewed and approved as exempt by the University of Missouri Institutional Review Board (IRB). The requirement to obtain informed consent was waived.

\section{Results}

In total, 997 patient encounters occurred at MedZou from August 2018 through March 2021. 773 of these encounters were held in-person before the onset of COVID-19 from August 2018 to February 2020. 264 encounters were held during the COVID-19 pandemic via telehealth from March 2020 through March 2021. Due to limited appointment availability after the onset of COVID-19, the average number of patient encounters per month declined from 38.6 encounters per month to 20.3 encounters per month, a $47.4 \%$ reduction in the number of patient encounters per month.

The sample was comprised of 330 unique patients, 309 of which were seen in-person before the onset of COVID-19 and were the subject of the main analysis. The remaining 21 patients were seen exclusively via telehealth for the first time after March 2020. In patients seen from September 2018 through February 2019, 51.2\% of patients returned to clinic in the following 6-month timeframe. Comparatively, patients seen from September 2019 through February 2020 returned at a rate of $29.6 \%$ in the 6 months following the onset of COVID-19, $X^{2}(1, \mathrm{~N}=280)=13.58, \mathrm{p}<0.001$.

Chi-squared bivariate analysis found that older patients were more likely to return for a telehealth visit during the COVID-19 pandemic. Of patients $<45$ years, $41.38 \%$ returned for a telehealth pandemic visit, versus $58.62 \%$ of patients 45 years of age and older $(\mathrm{p}=0.045)$ (Table 1). No other variables (sex, race, ethnicity, rurality) were associated with a return visit during COVID-19.

Results from logistic regression analysis confirmed the results of the bivariate analysis. Relative to younger patients, older patients were more likely to return for a telehealth visit after the onset of COVID-19 (OR 1.71, 95\% CI 1.02-2.85). The likelihood of a return visit did not differ by sex, race, ethnicity, or rurality (Fig. 1).

\section{Discussion}

The COVID-19 pandemic has had a far-reaching impact and our study offers insight into its effect on underserved and uninsured populations. Nationally, there were dramatic shifts in the ways in which care was delivered. Among 16.7 million Medicare enrollees, total visits in outpatient clinics reduced by $9.1 \%$, and the rates of telemedicine increased $2013 \%$ in the first 6 months of 2020 [23]. The impact on our SRFC was much greater, with a $47.4 \%$ reduction in outpatient clinic volume. It is important to characterize how these changes affected not only insured populations but uninsured populations as well, such as those receiving care from SRFCs.

Older patients ( $\geq 45$ years old) were more likely to return to clinic than younger patients $(<45$ years old $)$ after the onset of the COVID-19 pandemic. We know that there exists a generational technological divide between older and younger patients in that older patients are less likely to access the internet, use smartphones and have digital literacy [22, 24-26]. Therefore, this finding was somewhat surprising as our clinic had switched to telehealth-only available appointments in March 2021 and older patients were thought to be less likely to utilize technology, including 
Table 1 Patient demographics on return to clinic via telehealth during COVID-19 (bi-variate analysis)

\begin{tabular}{|c|c|c|c|c|c|c|c|c|}
\hline & \multicolumn{2}{|c|}{$\begin{array}{l}\text { Total patients } \\
(\mathrm{N}=309)\end{array}$} & \multicolumn{2}{|c|}{$\begin{array}{l}\text { Returned during } \\
\text { COVID-19 } \\
(\mathrm{N}=87)\end{array}$} & \multicolumn{2}{|c|}{$\begin{array}{l}\text { Did not return } \\
(\mathrm{N}=222)\end{array}$} & \multirow[t]{2}{*}{$\chi^{2}$} & \multirow[t]{2}{*}{ p-value } \\
\hline & $\mathrm{n}$ & $\%$ & $\mathrm{n}$ & $\%$ & $\mathrm{n}$ & $\%$ & & \\
\hline Age & & & & & & & 4.0169 & 0.045 \\
\hline$<45$ years & 156 & 50.49 & 36 & 41.38 & 120 & 54.05 & & \\
\hline$\geq 45$ years & 153 & 49.51 & 51 & 58.62 & 102 & 45.95 & & \\
\hline Sex & & & & & & & 1.1313 & 0.288 \\
\hline Female & 157 & 50.81 & 40 & 45.98 & 117 & 52.70 & & \\
\hline Male & 152 & 49.19 & 47 & 54.02 & 105 & 47.30 & & \\
\hline Race & & & & & & & 3.8119 & 0.149 \\
\hline Black or AA & 63 & 20.39 & 21 & 24.14 & 42 & 18.92 & & \\
\hline White & 199 & 64.40 & 58 & 66.67 & 141 & 63.51 & & \\
\hline Other & 47 & 15.21 & 8 & 9.20 & 39 & 17.57 & & \\
\hline Ethnicity & & & & & & & 2.7849 & 0.248 \\
\hline Hispanic & 15 & 4.85 & 2 & 2.30 & 13 & 5.86 & & \\
\hline Non-Hispanic & 269 & 87.06 & 80 & 91.95 & 189 & 85.14 & & \\
\hline Other & 25 & 8.09 & 5 & 5.75 & 20 & 9.01 & & \\
\hline Residence & & & & & & & 0.0007 & 0.979 \\
\hline Urban & 256 & 82.85 & 72 & 82.76 & 184 & 82.88 & & \\
\hline Rural & 53 & 17.15 & 15 & 17.24 & 38 & 17.12 & & \\
\hline
\end{tabular}

$A A$ African American

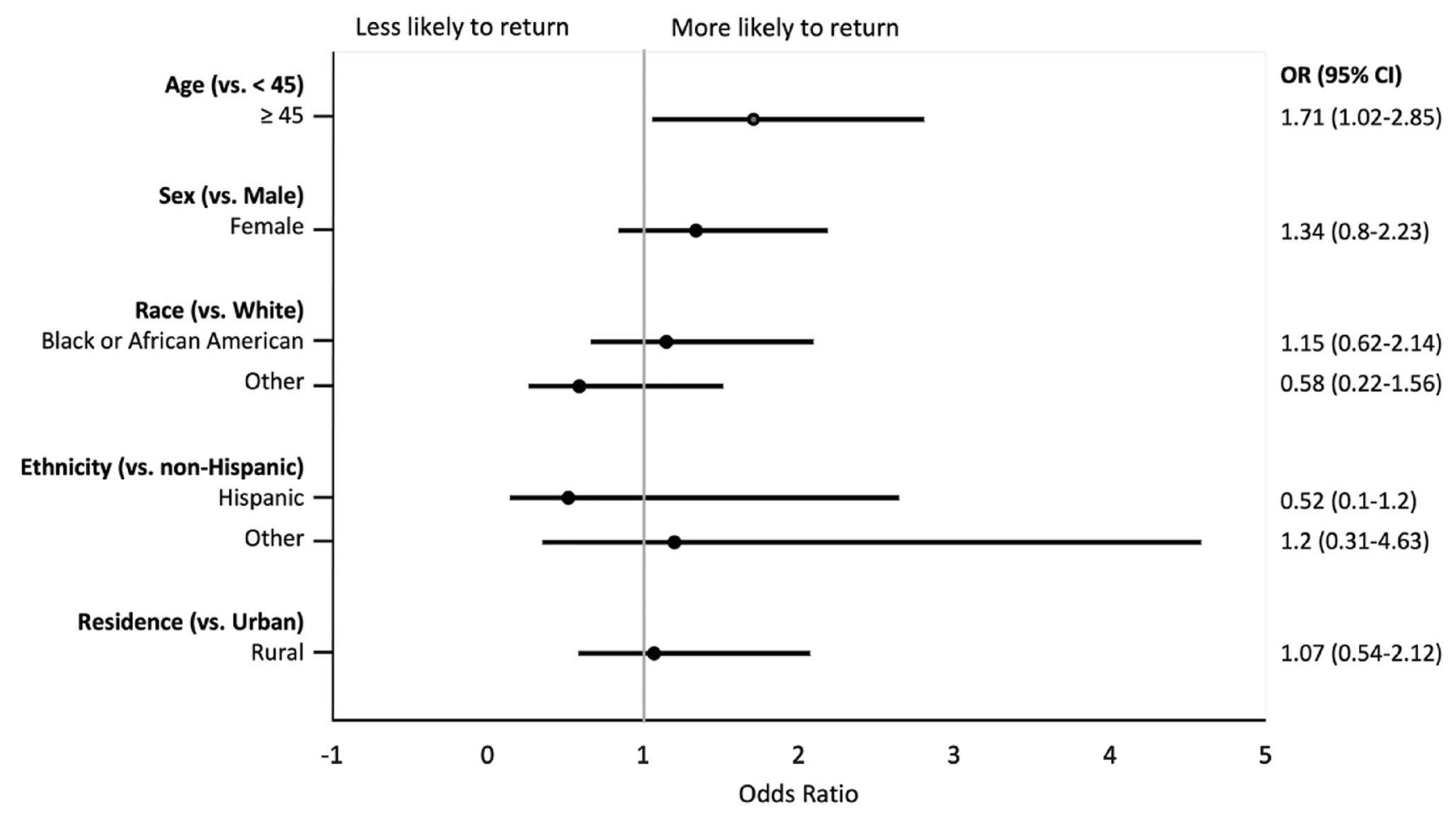

Fig. 1 Odds ratios (ORs) on patient return to clinic via telehealth during COVID-19 (multi-variate analysis)

telehealth. Telehealth was the only option for delivery of care in this uninsured population, and older patients are more likely to have chronic diseases necessitating more regular follow up [27]. The greater likelihood of chronic 
diseases requiring maintenance prescription medication, even after telehealth implementation, might account for these findings. Additionally, safety-net organizations, like this free-health clinic, often provide care for those adults who are not yet 65 years old and thus not yet eligible for Medicare. This is true of our clinic where there are only a handful of patients 65 years and older. Our findings may be explained by differences between the older adult population of 45-64 years old and an older adult population greater than or equal to 65 years old with respect to the technological divide.

COVID-19 has exacerbated racial and ethnic disparities [2-6]. We were very interested in understanding whether the COVID-19 pandemic and switch to telehealth had exacerbated racial and ethnic disparities at our SRFC. Our data indicated there was no difference in the likelihood to return to clinic based on race or ethnicity. This is consistent with other studies which showed no racial or ethnic disparities with telehealth utilization [22, 28, 29]. Lack of access to healthcare has become increasingly relevant as millions of individuals have recently lost health insurance because of pandemic-related unemployment [30]. We were pleased that telehealth and the COVID-19 pandemic did not create an added barrier that prevented access to care at MedZou for racial and ethnic underrepresented patients.

With respect to rurality, rural patients were just as likely to return to clinic as urban patients after the onset of the pandemic and telehealth implementation. Rural areas have a limited availability of broadband access, so we expected a decline in the return of our rural patients [31]. However, rural patients live farther away so it can be postulated that telehealth visits may save time in driving which could prove advantageous. Further research is indicated to identify whether telehealth serves as a way for rural residents to achieve greater access to care.

Our study had several limitations. First, the study comes from a single center with a small sample size. Our study might lack the power to detect disparities by race, ethnicity, sex, or rurality. Second, the findings related to age may be confounded by chronic illnesses and the need for prescription medications and refills. Controlling for the variables such as numbers of chronic conditions and prescription refills might further inform these findings. Finally, this study did not address the reason for each encounter. Further work comparing pandemic-related changes in both acute episodic care and care for chronic conditions in SRFCs may be valuable.

Telehealth has allowed many facilities, including our free clinic, to navigate the complex challenges presented by the COVID-19 pandemic. Although the pandemic and the primary utilization of telehealth during this time period did result in an overall decrease in our capacity for patient visits, we did not find that they were added barriers to care with regards to most patient demographics, including sex, race, ethnicity, and rurality.

Author Contributions MF, NH, ZR, and MS conceived the study; RP collected the data; ZR carried out the initial analysis; MF, NH, ZR, and MS wrote the manuscript; RP critically reviewed and revised the manuscript; MF, NH, ZR \& MS contributed equally as first authors. All authors approved of the final version of the manuscript.

Funding This project received no outside funding.

Code Availability Not applicable.

\section{Declarations}

Conflict of interest The authors have no conflicts of interest or competing interests to disclose.

Ethical Approval This study was reviewed and approved as exempt by the University of Missouri Institutional Review Board (IRB).

Consent to Participate The requirement to obtain informed consent was waived.

Consent for Publication Not applicable.

\section{References}

1. CDC. Trends in Number of COVID-19 Cases and Deaths in the US Reported to CDC, by State/Territory. Center for Disease Control: online.

2. Selden, T. M., \& Berdahl, T. A. (2020). COVID-19 and racial/ ethnic disparities in health risk, employment, and household composition. Health Affairs, 39(9), 1624-1632.

3. Webb Hooper, M., Nápoles, A. M., \& Pérez-Stable, E. J. (2020). COVID-19 and racial/ethnic disparities. JAMA, 323(24), 2466-2467.

4. Alcendor, D. J. (2020). Racial disparities-associated COVID-19 mortality among minority populations in the US. Journal of Clinical Medicine, 9(8), 2442.

5. Boserup, B., McKenney, M., \& Elkbuli, A. (2020). Disproportionate impact of COVID-19 pandemic on racial and ethnic minorities. The American Surgeon, 86(12), 1615-1622.

6. Garg, S., Kim, L., Whitaker, M., O'Halloran, A., Cummings, C., Holstein, R., et al. (2020). Hospitalization rates and characteristics of patients hospitalized with laboratory-confirmed coronavirus disease 2019-COVID-NET, 14 States, March 1-30, 2020. Morbidity and Mortality Weekly Report, 69(15), 458.

7. Smith, A. (2020). Missouri voters approve Medicaid expansion despite resistance from Republican leaders. In NPR kbia. https:// www.npr.org/sections/health-shots/2020/08/05/898899246/misso uri-voters-approve-medicaid-expansion-despite-resistance-fromrepublican-le

8. Han, X., Nguyen, B. T., Drope, J., \& Jemal, A. (2015). Healthrelated outcomes among the poor: Medicaid Expansion vs. NonExpansion States. PloS one, 10(12), e0144429. https://doi.org/10. 1371/journal.pone. 014442 
9. (AAMC), A.o.A.M.C. (2020). Important guidance for medical students on clinical rotations during the coronavirus (COVID-19) outbreak.

10. Using Telehealth to Care for Patients During the COVID-19 Pandemic. 2020 December 10, 2020 [cited 2020 December]. https:// www.aafp.org/family-physician/patient-care/current-hot-topics/ recent-outbreaks/covid-19/covid-19-telehealth.html

11. Getting started with telehealth. May 19, 2021 [cited 2020 November]. https://www.telehealth.hhs.gov/providers/getting-started/

12. Association, A.M. (2020). Telehealth implementation playbook (p. 128). American Medical Association.

13. Cook, E., Arboleda, B., Stewart, H., Nguyen, E., Shahin, A., Guerra, L., \& Gonzalez, E. (2021). Responding to COVID-19: Implementing a telemedicine program at a student-run free clinic. Telemedicine Reports, 2(1), 97-107.

14. Phan, R., Van Le, D., Nguyen, A., \& Mader, K. (2020). Rapid adoption of telehealth at an interprofessional student-run free clinic. PRiMER, 4, 23. https://doi.org/10.22454/PRiMER.2020. 241619

15. Ruiz Colón, G. D. M., Mulaney, B., Reed, R. E., Ha, S. K., Yuan, V., Liu, X., et al. (2021). The COVID-19 p+andemic as an opportunity for operational innovation at 2 student-run free clinics. Journal of primary care \& community health. https://doi.org/10. $1177 / 2150132721993631$

16. Zerihun, L. (2020). A telemedicine intervention for a student-run free clinic during COVID-19. Free Clinic Research Collective, $6(1)$.

17. Wojdyla, G., \& Hardy, K. (2020). KNIGHTS at home: The adaptation of a student-run clinic during COVID-19. Free Clinic Research Collective, 6(1)

18. Kim, J., Webb, N., Matos, N., Furuya, R., Kim, J., Webb, N., \& Matos, N. (2020). Learning from the COVID-19 pandemic: Designing and implementing a telehealth-in person hybrid care model. Free Clinic Research Collective, 6(1).

19. Kaplan, B. (2021). Access, equity, and neutral space: Telehealth beyond the pandemic. The Annals of Family Medicine, 19(1), 75-78.

20. Balaji, A., \& Clever, S. L. (2021). Incorporating medical students into primary care telehealth visits: Tutorial. JMIR Medical Education, 7(2), e24300.

21. Linggonegoro, D., Rrapi, R., Ashrafzadeh, S., McCormack, L., Bartenstein, D., Hazen, T. J., et al. (2021). Continuing patient care to underserved communities and medical education during the COVID-19 pandemic through a teledermatology student-run clinic. Pediatric Dermatology, 38(4), 977-979. https://doi.org/10. 1111/pde. 14653

22. Nguyen, O. T., Watson, A. K., Motwani, K., Warpinski, C., McDilda, K., Leon, C., et al. (2021). Patient-Level Factors Associated with Utilization of Telemedicine Services from a Free Clinic
During COVID-19. Telemedicine and e-Health. https://doi.org/10. 1089/tmj.2021.0102

23. Patel, S. Y., Mehrotra, A., Huskamp, H. A., Uscher-Pines, L., Ganguli, I., \& Barnett, M. L. (2021). Trends in outpatient care delivery and telemedicine during the COVID-19 pandemic in the US. JAMA Internal Medicine, 181(3), 388-391. https://doi.org/ 10.1001/jamainternmed.2020.5928

24. Nouri, S., Khoong, E. C., Lyles, C. R. \& Karliner, L. (2020). Addressing equity in telemedicine for chronic disease management during the Covid-19 pandemic. NEJM Catalyst Innovations in Care Delivery, 1(3), 1-13.

25. Din, H. N., McDaniels-Davidson, C., Nodora, J., \& Madanat, H. (2019). Profiles of a health information-seeking population and the current digital divide: Cross-sectional analysis of the 20152016 California Health Interview Survey. Journal of Medical Internet Research, 21(5), e11931. https://doi.org/10.2196/11931

26. Ye, S., Kronish, I., Fleck, E., Fleischutet, P. M., Homma, S., Masini, D., Moise, N. (2021). Telemedicine expansion during the COVID-19 pandemic and the potential for technology-driven disparities. Journal of General Internal Medicine, 36(1), 256-258. https://doi.org/10.1007/s11606-020-06322-y

27. He, Z., Bian, J., Carretta, H. J., Lee, J., Hogan, W. R., Shenkman, E., Charness, N. (2018). Prevalence of multiple chronic conditions among older adults in Florida and the United States: Comparative analysis of the OneFlorida data trust and national inpatient sample. Journal of Medical Internet Research, 20(4), e137. https:// doi.org/10.2196/jmir.8961

28. Graetz, I., Gordon, N., Fung, V., Hamity, C. \& Reed, M. E. (2016). The digital divide and patient portals: Internet access explained differences in patient portal use for secure messaging by age, race, and income. Medical Care, 54(8), 772-779.

29. Anthony, D. L., Campos-Castillo, C., \& Lim, P. S. (2018). Who isn't using patient portals and why? Evidence and implications from a national sample of US adults. Health Affairs (Millwood), 37(12), 1948-1954.

30. Boulware, L. E. (2020). Race disparities in the COVID-19 pandemic-Solutions lie in policy, not biology. JAMA Network Open, 3(8), e2018696.

31. Wilcock, A. D., Rose, S., Busch, A. B., Huskamp, H. A., UscherPines, L., Landon, B., \& Mehrotra, A. (2019). Association between broadband internet availability and telemedicine use. JAMA Internal Medicine, 179(11), 1580-1582. https://doi.org/ 10.1001/jamainternmed.2019.2234

Publisher's Note Springer Nature remains neutral with regard to jurisdictional claims in published maps and institutional affiliations. 